\title{
Persistent asthma has an accumulative impact on the loss of smell in patients with nasal polyposis*
}

\author{
Isam Alobid ${ }^{1,4,5}$, Sara Cardelus ${ }^{1}$, Pedro Benítez ${ }^{3}$, José Maria Guilemany ${ }^{1,4,5}$, Jordi \\ Roca-Ferrer ${ }^{4}$, Cesar Picado ${ }^{2,5}$, Manuel Bernal-Sprekelsen ${ }^{1, \S}$, Joaquim Mullol 1,4,5,
}

1 Rhinology Unit and Smell Clinic, Department of Otorhinolaryngology, Hospital Clinic, University of Barcelona, Spain

2 Department of Pneumology and Respiratory Allergy, Hospital Clínic, Department of Medicine, University of Barcelona, Spain

3 Department of Otorhinolaryngology, Hospital Sant Joan Despí - Moisès Broggi, Barcelona, Catalonia, Spain

4 Institut d'Investigacions Biomèdiques August Pi i Sunyer (IDIBAPS), Barcelona, Catalonia, Spain

5 Centro de Investigación Biomédica en Red de Enfermedades Respiratorias (CIBERES), Barcelona, Catalonia, Spain

SUMMARY

Background: Loss of sense of smell is one of the most frequent complaints in patients with nasal polyposis (NP). The aim of this study was to evaluate the impact of asthma and its severity on the sense of smell.

Material and methods: Patients with massive NP and healthy controls were included. More than half of patients presented with asthma. Olfactometry by Barcelona Smell Test 24, nasal symptoms score, nasal endoscopy, allergy study, and paranasal sinus CT scan were assessed.

Results: NP patients showed a significant impairment in smell detection, identification, and forced choice compared to the control. Asthmatics reported lower scores for detection, identification, and forced choice than non-asthmatic patients. Patients with persistent asthma had an increased impairment of sense of smell on detection, identification, and forced choice than patients with intermittent asthma. No significant differences were found between mild, moderate, and severe persistent asthmatics. Paranasal sinuses opacification was inversely correlated with smell detection, identification and forced choice.

Conclusion: These findings suggest that patients with NP have an impaired sense of smell, that asthma -particularly persistent asthma- has a further impact on sense of smell, and that loss of smell may be used as a clinical tool to identify the severity of both NP and asthma.

Key words: asthma, chronic rhinosinusitis, nasal polyposis, olfaction, smell loss

\section{INTRODUCTION}

Nasal polyposis (NP) is an inflammatory condition of unknown aetiology that is present in $2-4 \%$ of the European adult population ${ }^{(1,2)}$. NP is often associated with asthma and other respiratory diseases, such as cystic fibrosis, primary ciliary dyskinesia, and aspirin sensitivity (3) and idiopathic bronchiectasis ${ }^{(4)}$. Because the prevalence of NP is increased in severe asthma patients, the presence of NP in asthma patients is considered to be an important risk factor associated with asthma severity ${ }^{(5)}$. Smell loss is one of the most frequent complaints in patients with nasal polyps, often being the first symptom of the disease ${ }^{(6)}$, and having a negative impact on quality of life ${ }^{(7-9)}$. Olfactory deficits secondary to NP have traditionally been attributed to the inability of odorant molecules to access the olfactory mucosa, which is believed to remain histologically normal despite the aggressive inflammatory process in the respiratory regions of the nose ${ }^{(10,11)}$. However, the loss of smell associated with NP may be due to either mucosal obstruction or inflammation of the olfactory cleft ${ }^{(12,13)}$. 
The development of standardized olfactory tests that are available in every-day clinical practice has provided a more objective methodology to assess smell problems than the single symptom score ${ }^{(14)}$. Smell tests for clinical use have been developed in different countries, but no single test has gained general acceptance. The authors have validated the Barcelona Smell Test 24 (BAST-24) as a good and reliable method to assess olfactory function in clinical practice for the Spanish and Mediterranean population ${ }^{(15)}$.

We hypothesize that if the severity of asthma is associated with $\mathrm{NP}$, and NP is associated with the loss of the sense of smell, asthma severity should also be associated with smell disorders.

The aims of this study were: 1) to investigate the impact of NP on the sense of smell when compared with a healthy control group by using both symptoms and olfactometry (BAST-24); 2) to evaluate the added impact of asthma and its severity on the sense of smell; and 3) to evaluate whether nasal symptoms, paranasal sinus CT opacification, aspirin sensitivity, and atopy correlate with the loss of smell in asthma and its different levels of severity.

To our knowledge, this is the first published study, which evaluates the severity of asthma in patients with NP and its negative impact on the sense of smell by using a validated olfactory test. Hence, we can speculate on the use of the loss of smell as a predicting risk factor for severe asthma

\section{METHODS}

\section{Study population}

Patients with massive NP $(n=245)$ (score 2 or 3 of Lildholdt classification) treated between 2005 and 2009 were included in this study. Among them, 52.7\% $(\mathrm{n}=129)$ had asthma $(14.7 \%$ intermittent, $30.2 \%$ mild persistent, $33.3 \%$ moderate persistent, $21.7 \%$ severe persistent) while $24.1 \%(n=59)$ had aspirin sensitivity. The mean age was $50 \pm 13$ years (range $22-84$ years), $45 \%$ of them being female. The study was approved by the Ethics Committee of Hospital Clínic de Barcelona and signed informed consent was obtained from all patients.

\section{Inclusion and exclusion criteria}

The diagnosis of NP was based on the EP ${ }^{3} \mathrm{OS}$ criteria: nasal congestion in combination with nasal discharge, \pm facial pain/pressure, \pm reduction or loss of smell for more than 12 weeks, and presence of both nasal polyps by nasal endoscopy and mucosal changes within the ostiomeatal complex and/or paranasal sinuses by CT $\operatorname{scan}^{(1,2)}$. A washout period of 4 weeks for oral and intranasal steroids was applied to all patients before smell test assessment. Thus, none of the nasal polyp patients received oral or intranasal steroids treatment at the moment of smell assessment. Asthma treatment was not modified during the study and no patients received treatment with leukotriene antagonists.

\section{Study design}

Smell test (BAST-24), nasal symptoms score, nasal endoscopy, allergy study, and paranasal sinus CT scan were assessed in all NP patients included in the study.
Nasal symptoms: The intensity of nasal obstruction and loss of smell was scored as follows: 0 , no symptoms; 1 , mild symptoms; 2, moderate symptoms; and 3, severe symptoms.

BAST-24: As described previously, 24 odours were used in the BAST-24: a) 20 odours to assess the $1^{\text {st }}$ cranial nerve (olfactory): banana, gasoline, lemon, rose, onion, smoked, anise, coconut, vanilla, melon, mandarin, bitter almond, pineapple, cheese, strawberry, mushroom, eucalyptol, clove, turpentine, and peach; and b) 4 odours to asses the $5^{\text {th }}$ cranial nerve (trigeminal): formol, vinegar, ammonia, and mustard ${ }^{(15)}$. After being exposed for 5 seconds to an odorant, patients were asked by the researcher to answer a number of questions: 1) to test smell detection: "Did you smell anything?"; 2) to test smell identification: "Did you recognize this odour?"; and 3) to test smell forced choice: "Which of these four odours did you smell?". The healthy control group consisted of 120 healthy volunteers without subjective olfactory disturbance or nasosinusal disease, and with a mean age of $42 \pm 16$ years (ranging from 15 to 85 years), $50 \%$ of them being female.

Diagnosis of asthma: Asthma was evaluated by the same pneumologist using the Global Initiative for Asthma (GINA) guideline to classify the severity of asthma ${ }^{(16)}$. The four levels of severity were established by taking into account the medication needed to control the disease. Patients needing occasional bronchodilator rescue medication and normal lung function test were considered as mild intermittent asthma. Those patients requiring regular therapy with inhaled glucocorticoids (up to $800 \mu \mathrm{g}$ of budesonide or equivalent daily) were characterized as mild persistent asthma. Patients requiring inhaled GC associated with long-acting beta- 2 bronchodilators or montleukast were classified as moderate persistent asthma, and finally those patients requiring a high dose of inhaled GC (1000 $\mu \mathrm{g}$ or higher of budesonide or equivalent) associated with long-acting beta-2 bronchodilators were characterized as severe asthma ${ }^{(16)}$. In addition to the GINA criteria, we measured the blood eosinophilia (expressed as percentage of white blood cells) and serum total $\operatorname{IgE}(\mathrm{KU} / \mathrm{L})$. Asthmatic patients had increased blood eosinophilia $7.2 \pm 0.7$ and total $\operatorname{IgE} 200 \pm$ 45 than non-asthmatics ( $3.2 \pm 0.4$ and $171 \pm 50$ respectively), however, no significant differences were observed between asthma groups.

All patients were tested when asthma was clinically stable. In the case of patients with persistent asthma, the test was performed when there was no evidence that the patient was suffering an episode of exacerbation. Similarly, patients with intermittent asthma were explored during asymptomatic periods. Polyp size: Polyp size was scored in each nasal cavity (from 0 to 3) using nasal endoscopy: 0, no polyps; 1 , mild polyposis (small polyps not reaching the upper edge of the inferior turbinate); 2, moderate polyposis (polyps between the upper and lower edges of the inferior turbinate); 3 , severe polyposis (large polyps reaching the lower edge of the inferior turbinate $)^{(17)}$.

Atopy: A skin prick test with commercial extracts was performed using the most prevalent airborne allergens in our geographic area (Barcelona), including house dust mites, pollens, molds, and cat and dog dander. 
Imaging: A CT scan of paranasal sinuses was performed in all patients and was blindly staged by the same radiologist using the Lund-Mackay scoring system: 0 , no opacity; 1, partial opacity; and 2, total opacity for each of the sinuses. In addition, the ostiomeatal complex was scored 0 for no obstruction or 2 when obstructed. The system has a bilateral total score of $24^{(18)}$.

\section{Statistical analysis}

The data are presented as mean \pm SD (standard deviation). Unpaired Student's t test was used to compare NP outcomes with those of the healthy population. Pearson correlation coefficients were used to examine the association between the loss of smell using BAST-24 and gender, age, nasal symptoms, CT scan, atopy outcomes, and asthma severity. A p-value of less than 0.05 was considered statistically significant.

\section{RESULTS.}

\section{Nasal symptoms}

Patients scored the loss of sense of smell as the most intense complaint $(2.6 \pm 0.8)$ followed by nasal obstruction $(2.5 \pm$ 0.7). Asthmatic patients showed significantly higher scores for

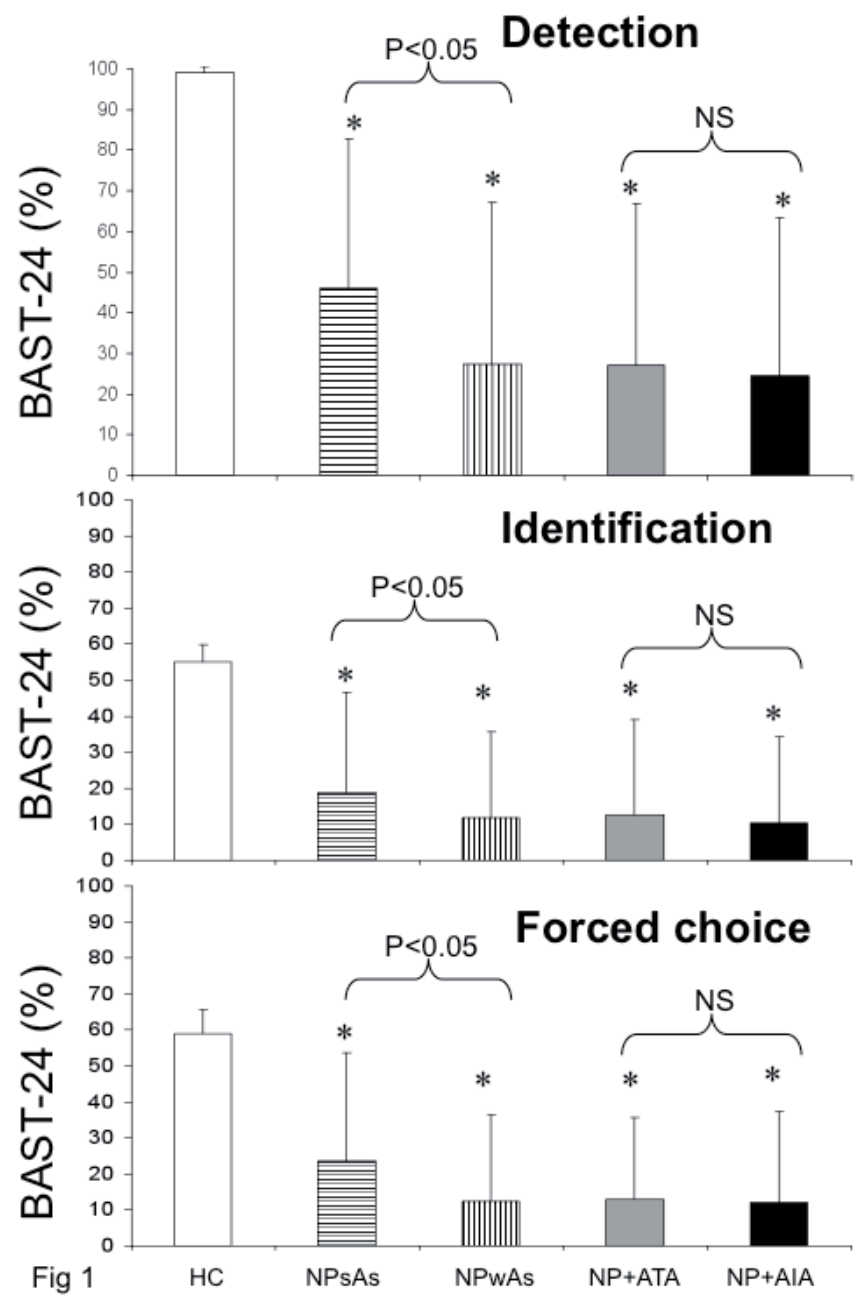

Figure 1. Smell test characteristics of patients with nasal polyposis (NP), Aspirin tolerant asthma (ATA), and Aspirin intolerant asthma $(\mathrm{AIA}) * \mathrm{p}<0.05$ compared to healthy population, $\dagger \mathrm{p}<0.05$ compared asthmatic to non-asthmatic patients. nasal obstruction and loss of sense of smell than non-asthmatic patients (Table 1). There was a negative mild but significant correlation between the loss of smell as a symptom and smell detection $(\mathrm{r}=-0.19 ; \mathrm{p}<0.01)$, identification $(\mathrm{r}=-0.20 ; \mathrm{p}<$ $0.01)$ and forced choice $(\mathrm{r}=-0.30 ; \mathrm{p}<0.0001)$ when assessed by BAST-24. No significant differences in nasal symptoms were observed between patients with aspirin-tolerant and aspirin-sensitive asthma or between atopic and non-atopic patients. Patients with persistent asthma reported a similar nasal obstruction but increased loss of smell compared to those with intermittent asthma (Table 1). In addition, there were no significant differences in nasal symptoms when comparing the different degrees of severity in persistent asthma.

\section{BAST-24}

Nasal polyp patients showed a significant $(\mathrm{p}<0.05)$ impaired smell detection $(36.7 \pm 43.5 \%)$, identification $(13.9 \pm 25.1 \%)$, and forced choice $(18.7 \pm 27.8 \%)$ than healthy volunteers $(99.2$ $\pm 1.5 \%, 54.7 \pm 4.9 \%, 72.2 \pm 6.8 \%$, respectively). No significant differences related to gender or age were found.
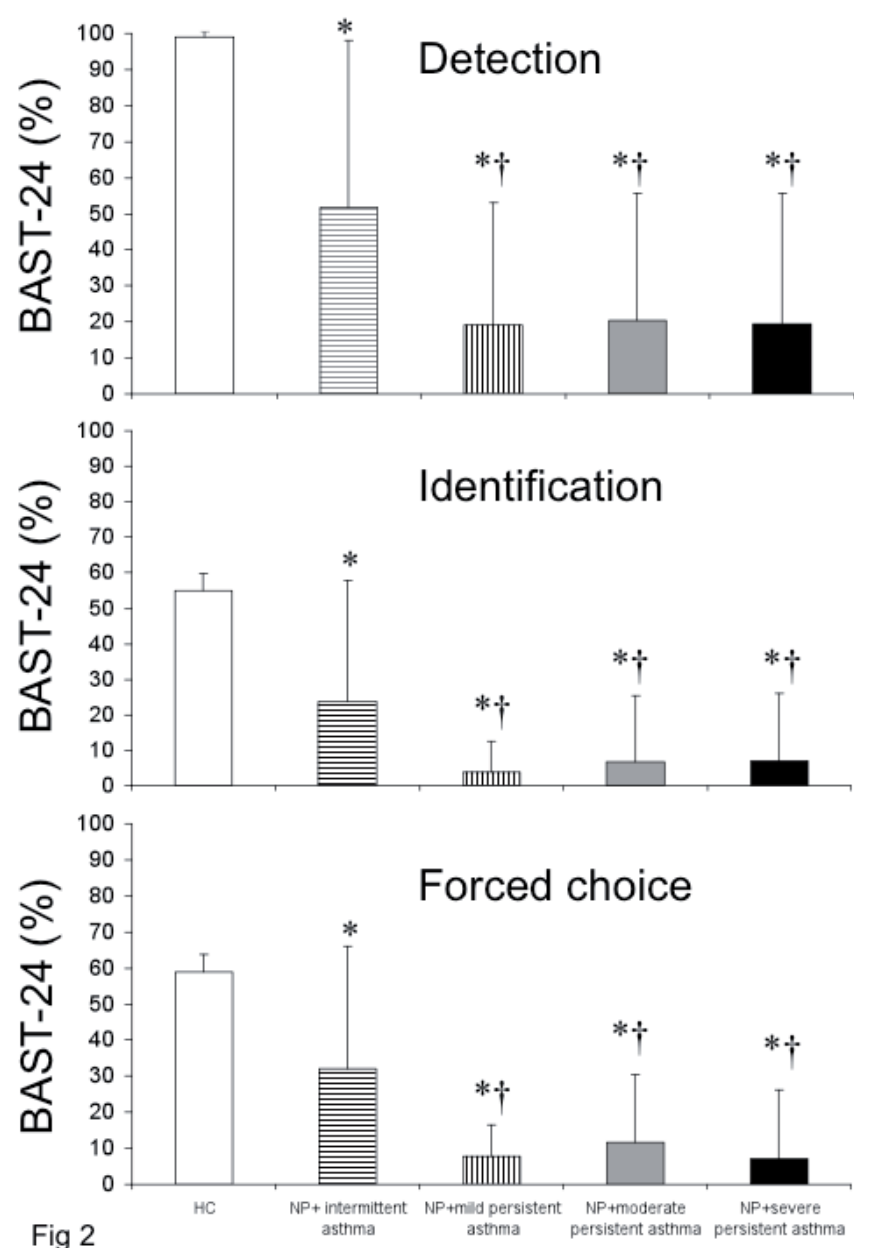

Figure 2. Smell test characteristics of patients with nasal polyposis (NP), ${ }^{*} \mathrm{p}<0.05$ compared to healthy population, $\uparrow \mathrm{p}<0.05$ compared persistent to intermittent asthmatic patients. 


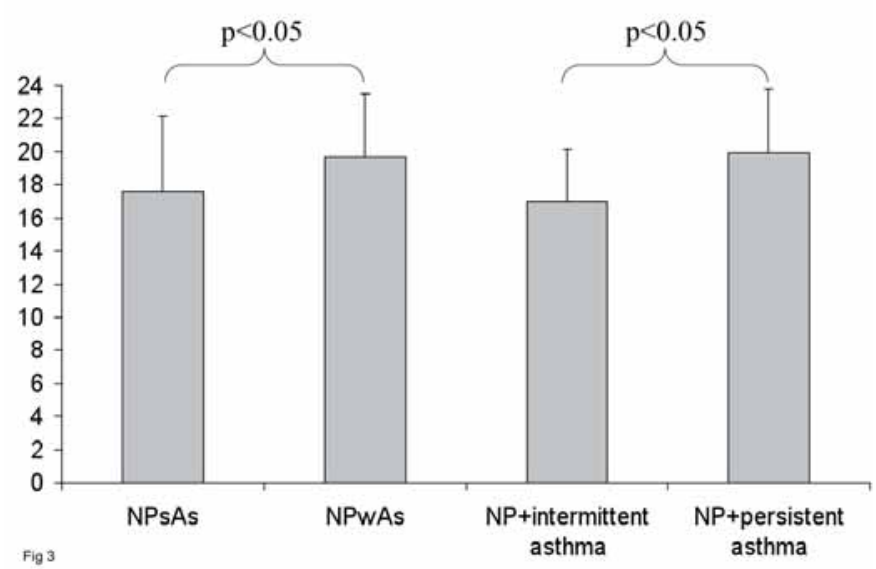

Figure 3. Paranasal sinuses CT scan opacification (Lund-Mackay score) of patients with nasal polyposis (NP).

Among nasal polyp patients, asthmatics reported lower scores $(\mathrm{p}<0.05)$ for detection $(27.3 \pm 39.9 \%)$, identification $(10.9 \pm$ $23.3 \%)$, and forced choice $(13.7 \pm 24.8 \%)$ than non-asthmatic patients $(47.1 \pm 45.1 \%, 17.4 \pm 26.6 \%, 24.2 \pm 29.9 \%$, respectively) (Figure 1). Patients with persistent asthma had a more impaired sense of smell on detection, identification, and forced choice than patients with intermittent asthma (Figure 2). No significant differences were found between aspirin-tolerant and aspirin-sensitive asthmatics (Figure 1) or when comparing the different levels of severity of persistent asthma (Figure 2).

\section{Polyp size}

All patients had moderate-sever NP (mean polyp size scores $=2.8 \pm 0.3$ ). No significant differences in polyp size were observed between asthmatics and non-asthmatics, with and without aspirin sensitivity, or between atopic and non-atopic patients. The polyp size score was not correlated with the loss of smell. This was probably because polyp size was very homogenous among the patients studied

No significant differences in polyp size were found between intermittent and persistent asthmatics (Table 1) or when comparing the degree severity in persistent asthma (data not shown).

\section{Atopy}

The skin prick test was positive in $32 \%$ of all nasal polyp patients, with no differences between males and females: house dust mites (18\%), pollens ( $22 \%)$, moulds $(9 \%)$, and animal dander $(14 \%)$. There were no significant differences between asthmatics and non-asthmatics, or between intermittent and persistent asthmatics. Using BAST-24 patients with NP and atopy showed no significant differences on smell detection (36.8 \pm $43.1 \%)$, identification $(18.5 \pm 31.8 \%)$, and forced choice $(15.3$ $\pm 25.9 \%$ ), compared to patients without atopy $(37.5 \pm 43.3 \%$, $12.9 \pm 25.3 \%, 18.9 \pm 27.2 \%$, respectively). Allergen type or multiple allergies had no accumulative negative impact on the loss of smell.

\section{Imaging}

The total CT scan score was $18.7 \pm 4.3$ for all nasal polyp patients. Compared with non-asthmatic patients, those with asthma were found to have a more extensive sinus disease, and this was more marked for persistent asthma than intermittent asthmatics (Figure 3). No significant differences in CT scan were observed between patients with aspirin-tolerant and aspirin-sensitive asthma or between atopic and non-atopic patients. A significant negative correlation $(\mathrm{p}<0.0001)$ was found between paranasal sinuses opacification and smell detection $(\mathrm{r}=-0.55)$, identification $(\mathrm{r}=-0.39)$, and forced choice $(\mathrm{r}$ $=-0.42)$ assessed by BAST -24 .

\section{DISCUSSION}

The main findings of our study using BAST-24 are: 1) nasal polyp patients have an impaired sense of smell when compared to the healthy population; 2) asthma and especially persistent asthma has an accumulative impact on the loss of smell in patients with NP; and 3) the loss of smell is correlated with paranasal sinus opacification. To our knowledge, this is the first study investigating the effect of asthma severity on the sense of smell in patients with NP by using both symptoms and subjective smell test.

Among the first studies performed in the 1950s to investigate olfaction in NP patients without objective sensory testing, Hotchkiss evaluated self-reported olfactory function in patients with nasal obstruction secondary to NP who also reported a loss of smell ${ }^{(19)}$. More recently, Kondo et al., found that the mean UPSIT score of the patients with rhinosinusitis/ NP was lower than that of controls. Detection and recognition thresholds showed a similar association and moderate correlations emerged between the odour test scores and the scores on a smell ability questionnaire ${ }^{(20)}$. Litvack et al., studied patients with chronic rhinosinusitis and found that $33 \%$ of patients showed normosmia, $45 \%$ hyposmia, and $22 \%$ anosmia. Patients with NP and over 65 years were at increased risk of hyposmia ${ }^{(21)}$. Vento et al., found that $46 \%$ of patients with long-standing NP had elevated olfactory thresholds above the upper limit of the $95 \%$ CI for a similarly aged reference group ${ }^{(22)}$. Cowart et al., assessed the olfactory threshold for phenylethyl alcohol in patients with symptoms of allergic rhinitis ${ }^{(23)}$. Olfactory thresholds were significantly higher in allergic patients, with $23.1 \%$ of the patients demonstrating a clinically significant loss of smell. Sinusitis or NP or both may underlie many cases of allergy-related hyposmia. Clinical or radiographic evidence of rhinosinusitis or NP or both in allergic patients was found to be associated with hyposmia: $14.3 \%$ of allergic patients with no associated rhinosinusitis showed hyposmia, whereas $42.9 \%$ of the allergy patients with associated rhinosinusitis did so ${ }^{(23)}$.

Using BAST-24, patients with NP and atopy showed no accumulative negative impact on the loss of smell. Rydzewski et al., found olfactory disorders in $21.4 \%$ of allergic patients who suffered from perennial rhinitis, seasonal rhinitis and bronchial 
asthma, performed according to Elsberg and Levy's method to determine both odour detection and odour identification thresholds. Of these, 13.8\% had anosmia and 7.6\% had hyposmia ${ }^{(24)}$. More recently, our group reported that patients with persistent allergic rhinitis have a moderate loss of smell (BAST24) with a higher impairment in those with self-reported hyposmia and moderate-to-severe persistent allergic rhinitis. The authors suggest a mixed etiology (obstruction/inflammation) as an explanation for smell disorder in allergic rhinitis ${ }^{(13)}$.

Both olfaction and nasal nitric oxide have been investigated and seem to be early markers of chronic rhinosinusitis ${ }^{(25)}$. Kern et al., analyzed 19 olfactory mucosa in patients with chronic rhinosinusitis, 9 of them with normal olfactory mucosa and olfactory function (UPSIT > 35). Ten patients demonstrated an influx of lymphocytes, macrophages, and eosinophils. Of these 10 patients, 7 had olfactory deficits as determined by UPSIT. These data suggest that the olfactory deficits may be the result of inflammatory changes within the olfactory mucosa in addition to any alteration in airflow to the olfactory cleft ${ }^{(26)}$. Soler et al., studied patients with chronic rhinosinusitis using the Smell Identification Test. Data showed that eosinophils correlate with objective disease severity as defined by CT, endoscopy, and smell test scores, even after controlling for comorbidities such as asthma, ASA intolerance, and NP ${ }^{(27)}$.

Our study has shown that asthma and, in particular, persistent asthma has an accumulative negative impact on the loss of smell in patients with NP. Bresciani et al., concluded that the frequency of rhinosinusitis in patients with mild-to-moderate or severe steroid-dependent asthma is similar; however, sinonasal involvement, as evaluated by clinical symptoms and CT scan, is significantly greater in patients with severe steroiddependent asthma than in those with mild-to-moderate asthma ${ }^{(28)}$. Bavbek et al., showed that NP was seen in $14 \%, 6 \%, 11 \%$, and $36 \%$ of mild intermittent and mild, moderate, and severe persistent asthma, respectively ${ }^{(29)}$. Ceylan et al., found that $54.3 \%$ of patients without NP, $63.2 \%$ with NP and $66.7 \%$ with Widal syndrome were at step 3 on the Global Initiative for Asthma scale of severity, while $1.9 \%, 15.8 \%$ and $33.3 \%$ were at step 4, respectively. They concluded that the presence of nasal polyps in asthma patients is associated with an increase in asthma severity ${ }^{(5)}$. Ehnhage et al., studied asthmatic patients with NP and identified lower subjective and objective olfactory outcomes (butanol threshold) compared to control norms ${ }^{(30)}$. Apter et al., demonstrated that the duration of nasal symptoms alone was not meaningfully correlated with the degree of olfactory loss. The allergy-immunology clinic patients were diagnosed as being normosmic and the taste and smell clinic patients as being hyposmic or anosmic with an olfactory loss that increased significantly with nasal-sinus disease severity (31). Staik囚niene et al., have recently reported that patients with chronic rhinosinusitis, NP, and asthma were characterized by older age, greater duration of nasal symptoms, a higher number of previous surgeries, more severe sinus disease on CT scan, bronchial obstruction, an incidence of allergic rhinitis, and sensitivity to house dust mite and mould allergens when compared to patients with chronic rhinosinusitis alone ${ }^{(32)}$. Litvack et al., showed that patients with NP, asthma, $\geq 65$ years old were at increased risk of anosmia ${ }^{(21)}$. In our study, we cannot demonstrate the risk factor of age on the sense of smell as Litvack et al., did by confirming that older patients are more susceptible to olfactory neuroepithelial injury and less able to recover from injury ${ }^{(21)}$.

Our study provides additional evidence that findings on the CT scan do not correlate with the severity of nasal symptoms as reported by other researches ${ }^{(33,34)}$. There was also no correlation between CT scan and atopy and polyp size ${ }^{(35)}$. However, Krouse reported that the degree of allergic reactivity is associated with the radiologic appearance of sinus mucosa on CT scan ${ }^{(36)}$. Bhattacharyya studied 161 patients with chronic rhinosinusitis and showed that for the absolute change in total symptom score, no statistically significant correlation with CT stage was demonstrated in any of the staging systems. Similarly, no significant correlation with CT stage was demonstrated with the other symptom domains ${ }^{(37)}$. Olfactory function was assessed with the 'Sniffin' Sticks' test and subjective perception was recorded with a visual analogue scale in 31 patients with NP. Disease in the upper meatus and the posterior portion of the middle meatus strongly affects olfactory function. Lund-Mackay scores had a significantly negative correlation with olfactory test results ${ }^{(38)}$. It should also be pointed out that our study presents results from a homogeneous group of patients with a specific nasal pathology (NP), in contrast to previous studies that have assessed patients with different nasal pathologies.

\section{CONCLUSION}

Our findings suggest that patients with NP have an impaired sense of smell, that asthma- particularly persistent asthma- has further impact on the loss of smell. Thus, the loss of smell on NP patients may be used as a clinical tool to identify asthma severity.

\section{REFERENCES}

1. Fokkens W, Lund V, Bachert C, et al. EAACI position paper on rhinosinusitis and nasal polyps executive summary. Allergy. 2005; 60: 583-601.

2. Fokkens W, Lund V, Mullol J. European Position Paper on Rhinosinusitis and Nasal Polyps Group. EP ${ }^{3} \mathrm{OS}$ 2007: European position paper on rhinosinusitis and nasal polyps 2007. A summary for otorhinolaryngologists. Rhinology. 2007; 45: 97-101.

3. Szczeklik A, Nizankowska E, Duplaga M. Natural history of aspirin-induced asthma. AIANE Investigators. European Network on Aspirin-Induced Asthma. Eur Respir J. 2000; 16: 432-436.

4. Guilemany JM, Angrill J, Alobid I, et al. United airways: the impact of chronic rhinosinusitis and nasal polyps in bronchiectasic patient's quality of life. Allergy. 2009; 64: 1524-1529.

5. Ceylan E, Gencer M, San I. Nasal polyps and the severity of asthma. Respirology. 2007; 12: 272-276.

6. Benítez P, Alobid I, de Haro J, et al. A short course of oral prednisone followed by long term intranasal budesonide is an effective treatment of severe nasal polyps. Comparative study of various methods of assessment. Laryngoscope. 2006; 116: 770-775.

7. Alobid I, Benítez P, Bernal-Sprekelsen M, et al. Nasal polyposis and its impact on quality of life. Comparison between the effects of medical and surgical treatments. Allergy. 2005; 60: 452-458. 
8. Alobid I, Benítez P, Bernal-Sprekelsen M, Guilemany JM, Picado C, Mullol J. The impact of asthma and aspirin sensitivity on quality of life of patients with nasal polyposis. Qual Life Res. 2005; 14: 789-793.

9. Alobid I, Bernal-Sprekelsen M, Mullol J. Chronic rhinosinusitis and nasal polyps. The role of generic and specific questionnaires on assessing its impact on patient s quality of life. Allergy. 2008; 63: 1267-1279.

10. Baroody F, Naclerio R. Allergic rhinitis. In: Getchell TV, Bartoshuk LM, Doty RL, Snow J, eds. Smell and Taste in Health and Disease. New York: Raven Press, 1991. p. 731-740.

11. Doty RL, Mishra A. Olfaction and its alteration by nasal obstruction, rhinitis, and rhinosinusitis. Laryngoscope. 2001; 111: 409423.

12. Guilemany JM, Angrill J, Alobid I, et al. United airways again: high prevalence of rhinosinusitis and nasal polyps in bronchiectasis. Allergy. 2009; 64: 790-797.

13. Guilemany JM, García-Piñero A, Alobid I, et al. Persistent allergic rhinitis has a moderate impact on the sense of smell, depending on both nasal congestion and inflammation. Laryngoscope. 2009; 119: 233-238.

14. Wrobel BB, Leopold DA. Clinical assessment of patients with smell and taste disorders. Otolaryngol Clin North Am. 2004; 37 : 1127-1142.

15. Cardesín A, Alobid I, Benítez P, et al. Barcelona Smell Test - 24 (BAST-24). Validation and smell characteristics in Spaniard healthy population. Rhinolgy. 2006; 44: 83-89.

16. Global Initiative for Asthma (GINA). National Asthma Education and Prevention Program: Expert Panel Report: Guidelines for the Diagnosis and Management of Asthma. Update on Selected Topics. National Institutes of Health, National Heart, Lung and Blood Institute. NIH publication No. 02-3659, Maryland, USA, 2002; 210-218

17. Lildholdt T, Rundcrantz H, Bende M, Larsen K. Glucocorticoid treatment for nasal polyps. The use of topical budesonide powder, intramuscular betamethasone, and surgical treatment. Arch Otolaryngol Head Neck Surg. 1997; 123: 595-600.

18. Lund VJ, Mackay I. Staging in rhinosinusitis. Rhinology. 1993; 31:183-184.

19. Hotchkiss WI. Influence of prednisone on nasal polyposis with anosmia. Ann Allergy. 1956; 24: 278-283.

20. Kondo H, Matsuda T, Hashiba M, Baba S. A study of the relationship between the T\&T olfactometer and the University of Pennsylvania Smell Identification Test in a Japanese population. Am J Rhinol. 1998; 12: 353-358

21. Litvack JR, Fong K, Mace J, James KE, Smith TL. Predictors of olfactory dysfunction in patients with chronic rhinosinusitis. Laryngoscope. 2008; 118: 2225-2230.

22. Vento SI, Simola M, Ertama LO, Malmberg CHO. Sense of smell in long- standing nasal polyposis. Am J Rhinol. 2001; 15: 159-163.

23. Cowart BJ, Flynn-Rodden K, McGeady SJ, Lowry LD. Hyposmia in allergic rhinitis. J Allergy Clin Immunol. 1993; 91: 747-751.

24. Rydzewski B, Pruszewicz A, Sulkowski WJ. Assessment of smell and taste in patients with allergic rhinitis. Acta Otolaryngol. 2000; 120: 323-326.

25. Landis BN, Lacroix JS. Olfactory function and nasal nitric oxide. Curr Opin Otolaryngol Head Neck Surg. 2009; 17: 18-22.
26. Kern RC. Chronic sinusitis and anosmia: pathologic changes in the olfactory mucosa. Laryngoscope. 2000; 110: 1071-1077.

27. Soler ZM, Sauer DA, Mace J, Smith TL. Relationship between clinical measures and histopathologic findings in chronic rhinosinusitis. Otolaryngol Head Neck Surg. 2009; 141: 454-461.

28. Bresciani M, Paradis L, Des Roches A, et al. Rhinosinusitis in severe asthma. J Allergy Clin Immunol. 2001; 107: 73-80.

29. Bavbek S, Celik G, Ediger D, et al. Severity and associated risk factors in adult asthma patients in Turkey. Ann Allergy Asthma Immunol. 2000; 85: 134-139.

30. Ehnhage A, Olsson P, Kölbeck KG, et al. Functional endoscopic sinus surgery improved asthma symptoms as well as PEFR and olfaction in patients with nasal polyposis. Allergy. 2009; 64: 762769.

31. Apter AJ, Gent JF, Rank ME. Fluctuating olfactory sensitivity and distorted odor perception in allergic rhinitis. Arch Otolaryngol Head Neck Surg. 1999; 125: 1005-1010.

32. Staik冈niene J, Vaitkus S, Japertiene LM, Ryskiene S. Association of chronic rhinosinusitis with nasal polyps and asthma: clinical and radiological features, allergy and inflammation markers. Medicina (Kaunas). 2008; 44: 257-265.

33. Wabnitz DA, Nair S, Wormald PJ. Correlation between preoperative symptom scores, quality-of-life questionnaires, and staging with computed tomography in patients with chronic rhinosinusitis. Am J Rhinol. 2005; 19: 91-96.

34. Stewart MG, Donovan DT, Parke RB Jr, Bautista MH. Does the severity of sinus computed tomography findings predict outcome in chronic sinusitis? Otolaryngol Head Neck Surg. 2000; 123: 81-84.

35. Alobid I, Benítez P, Valero A, et al. The impact of atopy, sinus opacification, and nasal patency on quality of life in patients with severe nasal polyposis. Otolaryngol Head Neck Surg. 2006; 134: 609-612.

36. Krouse JH. Computed tomography stage, allergy testing, and quality of life in patients with sinusitis. Otolaryngol Head Neck Surg. 2000; 123: 389-392.

37. Bhattacharyya N. Radiographic stage fails to predict symptom outcomes after endoscopic sinus surgery for chronic rhinosinusitis. Laryngoscope. 2006; 116: 18-22.

38. Konstantinidis I, Triaridis S, Printza A, Vital V, Ferekidis E, Constantinidis J. Olfactory dysfunction in nasal polyposis: correlation with computed tomography findings. ORL J Otorhinolaryngol Relat Spec. 2007; 69: 226-232.

\section{Isam Alobid}

Rhinology Unit and Smell Clinic

Department of Otorhinolaryngology

Hospital Clínic i Universitari

c/ Villarroel, 170

Barcelona 08036

Spain

Tel: 34- 932279872

E-mail: isamobid@hotmail.com 